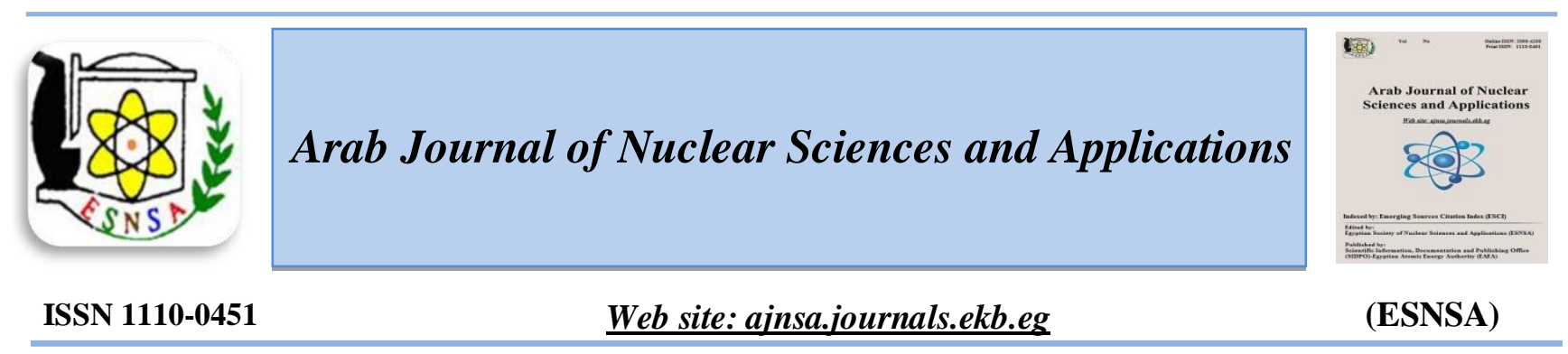

\title{
Therapeutic Role of Curcumin Loaded Magnetic Nanoparticles Against Gamma-Irradiation Hazards in Rats
}

\author{
H. A. Abdelmaksoud ${ }^{1}$; S. A. Hussein ${ }^{1}$; H. M. Saleh ${ }^{2}$; *W. F. Hassanin ${ }^{2}$., and E. I. Sobeh ${ }^{2}$ \\ ${ }^{I}$ Department of Biochemistry and clinical Biochemistry, Faculty of Veterinary Medicine, Benha University \\ ${ }^{2}$ Department of Biological Applications, Nuclear Research Center, Atomic Energy Authority, Egypt
}

\begin{abstract}
Received $11^{\text {st }}$ Jan 2019 Accepted $26^{\text {th }}$ May 2019

The present study has been carried out to investigate the possible therapeutic effect of curcumin loaded iron oxide nanoparticles (MNP-CUR) administered through intraperitoneal (i.p) injection after whole body gamma irradiation of rats. Whole body gamma irradiation of rats at $6 \mathrm{~Gy}$ (single dose) caused significant reduction in red blood cells count, hemoglobin levels, lymphocytes, neutrophils, and monocytes associated with highly significant elevation in malondialdehyde (MDA). The reduction in catalase (CAT) activity and the reduced levels of glutathione GSH were observed after three weeks postirradiation. Administration of MNP-CUR after irradiation resulted in a significant improvement of the hematological parameters andreduced changes in MDA, GSH and CAT levels were induced by gamma irradiation.The combination of MNP and CUR was found to be advantageous for the after-exposure treatment because the effect of radiation is stealth. An individual may spend days, weeks or even months exposed to radiation doses without becoming alarmed. Exposure to ionizing radiation is known to have an effect on the body cells, especially the blood cells and cause a marked decline in their viability and proliferation.
\end{abstract}

Keywords: Magnetic iron oxidenanoparticle, Gamma irradiation,Curcumin-loaded Magnetic nanoparticles, Herapeutic

\section{Introduction}

Ionizing radiation (IR) causes damage in biological tissues by exciting or ionizing their atoms and molecules. Depending on the radiation exposure dose, and the biochemical processes,the biological response to radiation damage may be prompt (the expression time may be within minutes to weeks after exposure)or delayed (the expression time may extend to several months or even years after exposure) [1]. With sufficient amount of radiation deposited energy within biological tissue, IR may induce physical symptomatology within minutes of exposure; the prodromal phase includes nausea, vomiting, and fatigue. These prodromal symptoms can be followed by a dramatic decrease in peripheral blood cell counts, as the blood renewal system of highly proliferating hemopoietic cells are known to be sensitive to IR [2]. The white blood cells show the most immediate response to IR by exhibiting a dramatic drop within $24 \mathrm{~h}$ (one day) following radiation exposure. In contrast, RBCs and platelets decline more gradually, over a longer time period $[3,4]$. Exposure to IR induces the production of reactive oxygen species (ROS), which include superoxide, hydroxyl radicals, singlet oxygen and hydrogen peroxide. These free radicals react with critical cellular components, such as DNA, RNA, proteins, and membranes, resulting in cell dysfunction and the initiation of apoptotic pathways leading to cell death $[5,6]$. Magnetic iron oxide nanoparticles (MNPs) are one of the most extensively investigated compounds, due to their excellent biocompatibility and ease of synthesis for a wide variety of applications [7-12]. Curcumin (CUR), (1,7-bis(4-hydroxy-3methoxyphenyl)- 1,6- heptadiene-3,5-dione), is a

Corresponding author: walaahassanin@ gmail.com

DOI: 10.21608/ajnsa.2019.7083.1167

(C) Scientific Information, Documentation and Publishing Office (SIDPO)-EAEA 
linear diarylheptanoid natural pigment isolated from the rhizome of Curcuma Longa [13]. CUR possesses a wide range of pharamacological activities, such as anticancer, anti-inflammatory, anti-oxidant and antimicrobial activity $[14,15]$. In addition, it has been also reported as a potential radio-protective agent [16, 17]. CUR exists in solution as a tautomeric mixture of keto and enol forms.

The keto-enol tautomerism is responsible for the free radical scavenging property of CUR $[18,19]$. The clinical use of CUR is of significant challenge due to its poor solubility in aqueous solutions, relatively low bioavailability, intense staining color and rapid metabolism[20] The aim of the present study is to investigate the therapeutic effect of curcumin-loaded magnetic nanoparticles (MNPCUR) in the treatment of the harmful effects of IR in rats.

\section{Materials and Methods}

Iron (II) chloride tetrahydrate $\left(\mathrm{FeCl}_{2}-4 \mathrm{H}_{2} \mathrm{O}\right)$, iron (III) chloride hexahydrate $\left(\mathrm{FeCl}_{3}-6 \mathrm{H}_{2} \mathrm{O}\right)$, polyethylene Glycol 600, and curcumin $\left(\mathrm{C}_{21} \mathrm{H}_{20} \mathrm{O}_{6}\right)$, were purchased from Sigma-Aldrich (USA). Ammonium hydroxide solution $\left(\mathrm{NH}_{4} \mathrm{OH}\right)$ was obtained from Merck (Germany). Iron oxide nanoparticles were prepared according to the method in Ref [21]. Loading CUR into the MNPs, wasconducted according to a previously reported protocol [22].

Produced MNP-CUR were characterized with transmission electron microscope (TEM),(EFI Netherland, Model Tecani G20), supertwin, double tilt, and Gun type $\mathrm{LaB}_{6}$. The applied voltage was $200 \mathrm{KV}$ and magnification reached 1000,000 X. The particle size and distribution in aqueous medium of the MNP-CUR formulation $(50 \mu \mathrm{L}$ of $500 \mu \mathrm{g} / \mathrm{mL}$ dispersed in $3 \mathrm{~mL}$ of distilled water and using ultrasonic path for 30 minutes). Spectral properties of freeze-dried CUR and MNP-CUR were also characterized and compared using Fourier transform infrared (FTIR), as powder in $\mathrm{KBr}$ at scanning speed of $4 \mathrm{~cm}^{-1}$ between $400-4000$ $\mathrm{cm}^{-1}$ (32 scans) on an illuminate IR TM FT-IR microscope (Smiths Detection, Danbury, CT).

Sixty adult male albino rats of pure strain ranging from 130-150g body weight were obtained from the animal house of the Animal Nutrition Research Unit in theNational Center for Radiation Research and technology, Egyptian Atomic Energy Authority. Animals were housed in especially designed cages (5 rats/ cage). All rats were kept under good conditions, allowed free access to tap water and pellet $\operatorname{diet}$ Ad libitum. The animals were divided into six groups(10 rats each): control group (Control), magnetic nanoparticles group (MNPs) which was injected i.p. with $1.5 \mathrm{mg} / \mathrm{kg}$ magnetite twice for three weeks, curcumin loaded magnetite group (CUR-MNPs) which was injected i.p. with $1.5 \mathrm{mg} / \mathrm{kg}$ curcumin loaded magnetite twice a week for three weeks, irradiated group (gamma radiation) exposed to sub-lethal (6Gy) whole body $\gamma$ - irradiation, Irradiated magnetic nanoparticles group (R+MNPs) which was exposed to sublethal dose (6Gy) gamma irradiation and then injected i.p. with magnetite NPs twice a week for three weeks and irradiated CUR-MNP Group (R+CUR-MNPs) which was exposed to sublethal dose (6 Gy) gamma irradiation and then injected i.p. with curcumin loaded magnetite NPs twice a week for three weeks. Whole body gamma irradiation was performed by Gamma- cell 40 (with cesium-137 gamma source) belonging to the National Center for Radiation Research Technology (NCRRT), Cairo, Egypt. Animals were exposed to irradiation 6 Gy as a single dose, at a dose rate of $0.957 \mathrm{Rad} / \mathrm{s}$. Blood samples were taken weekly from the retro-orbital venous plexus. Each blood sample was collected into tubes containing heparin for complete blood picture. At the end of the experiment after three weeks animals in all groups were sacrificed by decapitation and their liverswere excised, then perfused with cold saline to exclude the blood cells and then blotted on filter paper, and stored at $20^{\circ} \mathrm{C}$ for subsequent biochemical analysis (MDA, CAT and GSH). Complete blood counting (CBC) was determined by cell counter fully automated (Sysmex, Japan) [23]. Liver glutathione concentration was determined according to the method described by [24]. Catalase activity was determined according to the method described by [25]. MDA concentration was determined according to the method described by [26]. All results were expressed in mean \pm SE. Statistical analysis was performed using statistical package for the social science for windows (SPSS, version 11.0, Chicago, IL, USA). The data were analyzed by one-way analysis of variance (ANOVA). 


\section{Results}

The TEM image in Fig.(1) revealed an average size of 10-20 nm for CUR-MNPs. The TEM image of specific areas showed particles with sphericallike shapes, although for a few particles with the largest diameters observed faceted particles. The latter shape is probably related to the high crystallinity of the particles reflecting the cubic crystal habit of the $\mathrm{Fe}_{3} \mathrm{O}_{4}$ phase.

Fourier transform infrared (FTIR) spectra of CUR, CUR-MNP, and MNPs are illustrated in Fig.(2a). The transmittance of FTIR results is converted to absorption coefficient in the unit of $\mathrm{g}^{-1}$ was obtained from the relation

$\alpha=\frac{1}{m} \ln \frac{100}{T}$
Where $\mathrm{m}$ is the absolute mass of the curcumin in the $\mathrm{KBr}$ pellet and $\mathrm{T}$ is the infrared transmittance (\%). Using this technique, the normalized infrared absorption was calculated and presented in Fig.(2b); the comparison between the absorption curves showed the following:

1. A characteristic band at around $3443 \mathrm{~cm}^{-1}$ which is attributed to the $\mathrm{O}-\mathrm{H}$ stretching vibration of curcumin while, in the loaded system, in the presence of PEG assisted nanoparticles, the $\mathrm{O}-\mathrm{H}$ stertching vibration shifted to $3423.4 \mathrm{~cm}^{-1}$. The O-H absorption around $3443 \mathrm{~cm}^{-1}$, nearly, did not change on loading the CUR on MNPs. Similarly, the absorptions at $2880 \mathrm{~cm}^{-1}, 1616 \mathrm{~cm}^{-1}$, and around $1100 \mathrm{~cm}^{-1}$. This observation indicates that the antioxidant property of CUR did not change by legation to MNPs.

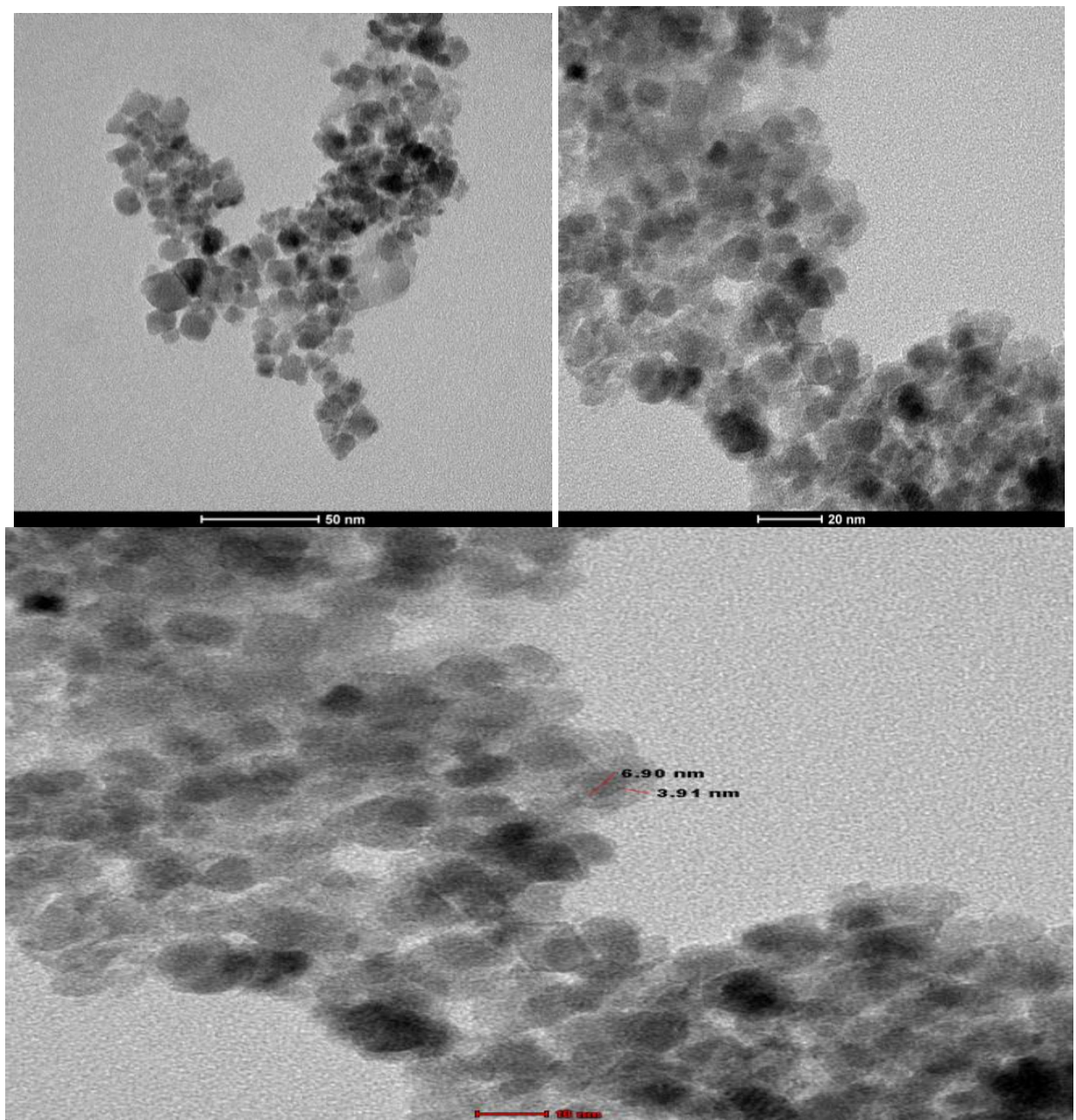

Figure (1):TEM image of curcumin loaded Fe3O4 magnetic nanostructurestransmission electron microscopy. The microstructural features of the MNP-CUR were studied using TEM 
2. The apparent increase in the peak intensity is associated with increasing the wide bands at $1360 \mathrm{~cm}^{-1}$ and $990 \mathrm{~cm}^{-1}$. These bands may be associated with Mono-Alkyl (vinyl group of reduced PEG at the magnetite surface, i.e. R$\mathrm{CH}=\mathrm{CH}_{2}$ ). Furthermore, shifting of the strong band at $1655 \mathrm{~cm}^{-1}$ of CUR to 1649 $\mathrm{cm}^{-1}$ in conjugated system indicated the participation of the dione moiety in conjugation. Moreover, the shifting of the stertching vibrational band from $1027.8 \mathrm{~cm}^{-1}$ to $1030.7 \mathrm{~cm}^{-1}$ in the loaded system is an indication of interaction of oxygen of DMF with MNPs.

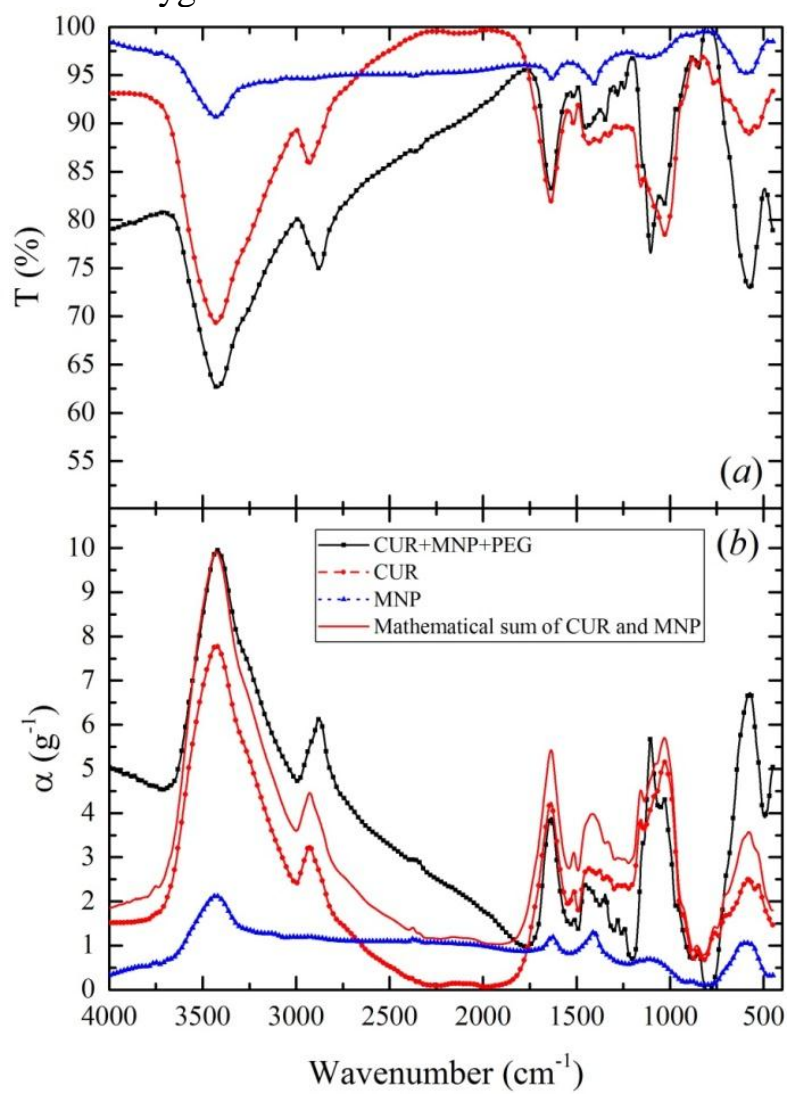

Figure (2):FTIR of dried curcumin (CUR), magnetite (MNP), and CUR-MNP. Part a) transmission mode, Part b) mass absorption coefficient in the units of $\mathrm{g}^{-1}$
As shown in Table (1) and Figs. (3,4,5 and 6), there was a significant decline, where the decision $\mathrm{p}$ value where $\mathrm{P}<0.05$, in WBCs count, lymphocyte, Neutrophil and Monocyte in all irradiated groups in the first week (T1) post 6Gy $\gamma$ irradiation. In the later samples of the second week (T2) and the third week (T3), unlike radiation group, there was gradual recovery in the levels of all WBCs types of R+MNPs and R+CURMNPs groups which became in the range of the levels of the control group. The best improvement was in R+CURMNPs group which recorded $6.7 \pm 0.46$ $\mathrm{x} 10^{3}, 5.2 \pm 0.03 \times 10^{3}, 0.4 \pm 0.005 \times 10^{3}, 0.5 \pm 0.05 \times 10^{3}$ for the values of WBCs count, lymphocyte, Neutrophil and Monocyte, respectively, with no significant difference compared to the control group after three weeks of irradiation.

The CBC parameters levels in the CUR-MNPs group showed a significant $(\mathrm{P}<0.05)$ improvement in the values of RBCs and $\mathrm{Hb}$ levels compared to control and MNPs groups, as demonstrated in Table (2) and Figs. (7,8,9, and 10). For this reason, the CBC values of the CUR-MNPs group was used as reference values for changes in CBC of irradiated groups. There was a significant decline in the RBC, Hb level, HCT\% and platelet of the irradiated groups compared to CUR-MNPs $(\mathrm{P}<0.05)$ in samples of the first week post $6 \mathrm{~Gy} \gamma$ irradiation. This decline continued in the radiation group in the later samples obtained at the secondand third-weeks post irradiation. On the other hand, recovery in CBC parameters levels was recorded in the $\mathrm{T} 2$ and $\mathrm{T} 3$ samples obtained from $\mathrm{R}+\mathrm{MNPs}$ and $\mathrm{R}+\mathrm{CURMNPs}$ groups. As in WBCs results, the improvements were highest in R+CURMNPs which reached near the CBC values of CUR-MNPs group $(\mathrm{P}<0.05)$. The recorded data in Table (3) and Figs. (11 and 12) showed that at the first week (T1), second week (T2) and third week (T3) post 6Gy $\gamma$-irradiation, the activity of the antioxidant enzymes; CAT, GSH in the liver tissue in irradiated group was significantly decreased ( $\mathrm{P}$ $<0.05$ ), as compared the control group, However, the liver tissue MDA, in the irradiated group, was significantly increased $(\mathrm{P}<0.05)$ when compared to the control group. Conversely, the irradiated rats treated with MNPs and CUR-MNPs showed a significant improvement in the antioxidant status via enhancement of the antioxidant enzymes activities (GSH, CAT). 
Table (1): the effect of MNP and MNP-CUR on the recovery of different types of white blood cells in in samples obtained from animals exposed to $6 \mathrm{~Gy}$ whole body $\gamma$-Irradiation at three weeks of study

\begin{tabular}{|c|c|c|c|c|c|c|c|c|c|c|c|c|}
\hline \multirow[b]{3}{*}{ Groups } & \multicolumn{12}{|c|}{ Post irradiation (Therapeutic experiment) } \\
\hline & \multicolumn{3}{|c|}{ WBCs $\left(\mathbf{x 1 0}^{3}\right)$} & \multicolumn{3}{|c|}{ Lymphocyte $\left(\mathrm{x10}^{3}\right)$} & \multicolumn{3}{|c|}{ Neutrophil $\left(\mathbf{x 1 0}^{3}\right)$} & \multicolumn{3}{|c|}{ Monocyte $\left(\mathbf{x 1 0}^{3}\right)$} \\
\hline & T1 & T2 & T3 & T1 & T2 & T3 & T1 & T2 & T3 & T1 & T2 & T3 \\
\hline Control & $\begin{array}{c}7.2^{\mathrm{a}} \\
\pm \\
0.13\end{array}$ & $\begin{array}{c}7.1^{\mathrm{a}} \\
\pm 0.13\end{array}$ & $\begin{array}{c}7.8^{\mathrm{a}} \pm \\
0.37\end{array}$ & $\begin{array}{c}5.8^{\mathrm{a}} \\
\pm 0.03\end{array}$ & $\begin{array}{l}5.9^{\mathrm{a}} \pm \\
0.358\end{array}$ & $\begin{array}{c}6.4^{\mathrm{a}} \\
\pm 0.202\end{array}$ & $\begin{array}{l}0.3^{\text {abc }} \\
\pm 0.14\end{array}$ & $\begin{array}{l}0.3^{\mathrm{ab}} \pm \\
0.016\end{array}$ & $\begin{array}{c}0.5^{\mathrm{ab}} \\
\pm 0.050\end{array}$ & $\begin{array}{c}0.4^{\mathrm{bc}} \\
\pm 0.070\end{array}$ & $\begin{array}{l}0.5^{a b} \pm \\
0.016\end{array}$ & $\begin{array}{c}0.7^{\mathrm{a}} \\
\pm 0.01\end{array}$ \\
\hline MNPs & $\begin{array}{c}7.6^{\mathrm{a}} \\
\pm \\
0.23 \\
\end{array}$ & $\begin{array}{r}7.5^{\mathrm{a}} \\
\pm 0.2\end{array}$ & $\begin{array}{c}7.7^{\mathrm{a}} \\
\pm \\
\mathbf{0 . 2 1} \\
\end{array}$ & $\begin{array}{c}5.8^{\mathrm{a}} \\
\pm \mathbf{0 . 0 3 2}\end{array}$ & $\begin{array}{l}5.7^{\mathrm{a}} \\
\mathbf{\pm 0} \\
031 \\
\end{array}$ & $\begin{array}{c}6^{\text {ab }} \\
\pm \\
0.033 \\
\end{array}$ & $\begin{array}{c}0.5^{\mathrm{a}} \\
\pm 0.064\end{array}$ & $\begin{array}{c}0.4^{\mathrm{ab}} \\
\pm 0.040\end{array}$ & $\begin{array}{c}0.5^{\text {abc }} \\
\pm 0.062\end{array}$ & $\begin{array}{c}0.4^{\mathrm{dc}} \\
\pm 0.011\end{array}$ & $\begin{array}{c}0.5^{\mathrm{ab}} \\
\pm 0.015\end{array}$ & $\begin{array}{c}0.6^{\mathrm{a}} \\
\pm 0.01\end{array}$ \\
\hline CURMNPs & $\begin{array}{c}7.6^{\mathrm{a}} \\
\pm \\
0.34\end{array}$ & $\begin{array}{c}7.3^{\mathrm{a}} \\
\pm \\
\mathbf{0 . 3 4}\end{array}$ & $\begin{array}{c}6.8^{\mathrm{a}} \\
\pm 0.60\end{array}$ & $\begin{array}{c}6.5^{\mathrm{a}} \\
\pm 0.02\end{array}$ & $\begin{array}{c}6.3^{\mathrm{a}} \\
\pm 0.010\end{array}$ & $\begin{array}{c}5.8^{\mathrm{a}} \\
\pm \mathbf{0 . 0 2 0}\end{array}$ & $\begin{array}{c}0.4^{\mathrm{ab}} \\
\pm 0.062\end{array}$ & $\begin{array}{c}0.5^{\mathrm{a}} \\
\pm 0.028\end{array}$ & $\begin{array}{c}0.2^{\mathrm{d}} \\
\pm \mathbf{0 . 0 3 7}\end{array}$ & $\begin{array}{c}0.7^{\mathrm{a}} \\
\pm \\
0.085\end{array}$ & $\begin{array}{c}0^{0.6^{a}} \\
\pm 0.033\end{array}$ & $\begin{array}{c}0.6^{\mathrm{a}} \\
\pm \\
0.09\end{array}$ \\
\hline Radiation & $\begin{array}{c}1.2^{\mathrm{d}} \\
\pm \\
0.20 \\
\end{array}$ & $\begin{array}{c}1.3^{d} \\
\pm 0.20\end{array}$ & $\begin{array}{c}2.4^{b} \\
\pm 0.10\end{array}$ & $\begin{array}{c}0.5^{c} \\
\pm 0.71\end{array}$ & $\begin{array}{c}0.7^{c} \\
\pm 0.72\end{array}$ & $\begin{array}{c}1.4^{c} \\
\pm 0.12\end{array}$ & $\begin{array}{l}0.02^{d} \\
\pm 0.33\end{array}$ & $\begin{array}{c}0.03^{\mathrm{c}} \\
\pm \mathbf{0 . 0 3 3}\end{array}$ & $\begin{array}{c}0.02^{\mathrm{d}} \\
\pm \\
\mathbf{0 . 0 3 7} \\
\end{array}$ & $\begin{array}{l}0.03^{\mathrm{e}} \\
\pm \mathbf{0 . 0 1}\end{array}$ & $\begin{array}{c}0.02^{c} \\
\pm 0.054\end{array}$ & $\begin{array}{c}0.1^{b} \\
\pm \\
0.04 \\
\end{array}$ \\
\hline R+MNPs & $\begin{array}{c}2.2^{\mathrm{c}} \\
\pm \\
0.08\end{array}$ & $\begin{array}{c}3.5^{\mathrm{c}} \\
\pm \\
0.08\end{array}$ & $\begin{array}{c}5.9^{\mathrm{a}} \\
\pm \\
0.67\end{array}$ & $\begin{array}{c}1.2^{\mathrm{b}} \\
\pm 0.32\end{array}$ & $\begin{array}{c}2.1^{b} \\
\pm 0.42\end{array}$ & $\begin{array}{l}4.1^{\mathrm{abc}} \\
\pm 4.3\end{array}$ & & $\begin{array}{c}0.13^{\text {bc }} \\
\pm 0.033\end{array}$ & $\begin{array}{c}0.3^{\mathrm{dc}} \\
\pm 0.040\end{array}$ & $\begin{array}{c}0.07^{\text {de }} \\
\pm 0.033\end{array}$ & $\begin{array}{c}0.2^{b} \\
\pm 0.0251\end{array}$ & $\begin{array}{c}0.4^{\mathrm{a}} \\
\pm \\
0.05\end{array}$ \\
\hline $\begin{array}{c}\text { R+ } \\
\text { CURMNPs }\end{array}$ & $\begin{array}{c}3.6^{6} \\
\pm \\
0.08 \\
\end{array}$ & $\begin{array}{c}5.0^{b} \\
\pm \\
0.08 \\
\end{array}$ & $\begin{array}{c}6.7^{\mathrm{a}} \\
\pm \\
0.46 \\
\end{array}$ & $\begin{aligned} & 2^{b} \\
\pm & 0.33\end{aligned}$ & $\begin{array}{c}3.1^{b} \\
\pm 0.04\end{array}$ & $\begin{array}{l}5.2^{\mathrm{ab}} \\
\pm 0.03\end{array}$ & $\begin{array}{c}2.7^{\text {bdc }} \\
\pm \\
\mathbf{0 . 0 3 3} \\
\end{array}$ & $\begin{array}{c}0.2^{\mathrm{ab}} \\
\pm 0.051\end{array}$ & $\begin{array}{c}0.4^{\text {bdc }} \\
\pm 0.005\end{array}$ & $\begin{array}{c}0.1^{\text {dce }} \\
\pm 0.005\end{array}$ & $\begin{array}{c}0.4^{\mathrm{ab}} \\
\pm 0.05\end{array}$ & $\begin{array}{c}0.5^{\mathrm{a}} \\
\pm \\
0.05 \\
\end{array}$ \\
\hline
\end{tabular}

Data are presented as means \pm S.E $\quad$ S.E.: Standard Error

Means values within the same column carrying different superscript letters are significantly different $(\mathbf{P}<0.05)$

Table(2): the effect of MNP and MNP-CUR on the recovery of different types of white blood cells in in samples obtained from animals exposed to $6 \mathrm{~Gy}$ whole body $\gamma$-Irradiation at three weeks of study

\begin{tabular}{|c|c|c|c|c|c|c|c|c|c|c|c|c|}
\hline \multirow{3}{*}{$\begin{array}{c}\text { Parameter } \\
\text { Groups }\end{array}$} & \multicolumn{12}{|c|}{ Post irradiation (Therapeutic experiment) } \\
\hline & \multicolumn{3}{|c|}{ RBCs } & \multicolumn{3}{|c|}{$\mathbf{H b}$} & \multicolumn{3}{|c|}{ HCT } & \multicolumn{3}{|c|}{ Platelet } \\
\hline & T1 & $\mathbf{T 2}$ & T3 & T1 & $\mathbf{T 2}$ & T3 & T1 & $\mathbf{T 2}$ & T3 & T1 & $\mathbf{T 2}$ & T3 \\
\hline Control & $\begin{array}{c}5.9 \mathrm{~d}^{\mathrm{c}} \\
\pm 0.2\end{array}$ & $\begin{array}{l}5.80^{b} \\
\pm 0.11\end{array}$ & $\begin{array}{c}5.8^{\mathrm{b}} \\
\pm 0.1 \\
1 \\
\end{array}$ & $\begin{array}{l}12.72^{\mathrm{c}} \\
\pm 0.34\end{array}$ & $\begin{array}{l}12.1 b^{c} \\
\pm 0.23\end{array}$ & $\begin{array}{r}12.2 \mathrm{a} \\
\mathbf{b}^{\mathrm{c}} \\
\pm 0.31 \\
\end{array}$ & $\begin{array}{c}37.1^{\mathrm{a}} \\
\pm 1.8 \\
1 \\
\end{array}$ & $\begin{array}{c}37.9^{\mathrm{a}} \\
\pm 1.2 \\
2 \\
\end{array}$ & $\begin{array}{r}37.8^{\mathrm{a}} \\
\pm 0.05\end{array}$ & $\begin{array}{l}711.2^{\mathrm{a}} \\
\pm 25.1\end{array}$ & $\begin{array}{c}710^{a} \\
\pm 25.4\end{array}$ & $\begin{array}{c}729.8^{\mathrm{a}} \\
\pm 18.8\end{array}$ \\
\hline MNPs & $\begin{array}{l}6.45 b^{c} \\
\pm 0.22\end{array}$ & $\begin{array}{l}6.33^{\mathrm{ab}} \\
\pm 0.11\end{array}$ & $\begin{array}{c}\mathbf{6 . 3 3}_{\mathrm{b}}^{\mathrm{a}} \\
\pm 0.1 \\
1\end{array}$ & $\begin{array}{c}12.72^{b} \\
c \\
\pm 0.32\end{array}$ & $\begin{array}{l}12.6^{\mathrm{ab}} \\
\pm 0.39\end{array}$ & $\begin{array}{l}12.0^{\mathrm{bc}} \\
\pm 0.25\end{array}$ & $\begin{array}{c}37.2^{\mathrm{a}} \\
\pm \\
0.58\end{array}$ & $\begin{array}{c}38.1^{\mathrm{a}} \\
\pm 0.9 \\
6\end{array}$ & $\begin{array}{r}37.5^{\mathrm{a}} \\
\pm 1.13\end{array}$ & $\begin{array}{r}659.2^{\mathrm{a}} \\
\pm 19.4\end{array}$ & $\begin{array}{l}676.5^{\mathrm{a}} \\
\pm 27.5\end{array}$ & $\begin{array}{c}674.2^{\mathrm{a}} \\
\pm 120 \\
9\end{array}$ \\
\hline $\begin{array}{c}\text { CURMNP } \\
\mathrm{s}\end{array}$ & $\begin{array}{c}7.3^{\mathrm{a}} \\
\pm 0.09\end{array}$ & $\begin{array}{c}6.80^{\mathrm{a}} \\
\pm 0.05 \\
7\end{array}$ & $\begin{array}{c}6.80^{\mathrm{a}} \\
\pm 0.0 \\
5\end{array}$ & $\begin{array}{l}13.85^{\mathrm{a}} \\
\pm 0.06\end{array}$ & $\begin{array}{c}13.9^{\mathrm{a}} \\
\pm 0.14 \\
9\end{array}$ & $\begin{array}{r}13.6^{\mathrm{a}} \\
\pm 0.30\end{array}$ & $\begin{array}{c}39.7^{\mathrm{a}} \\
\pm 1.1 \\
5\end{array}$ & $\begin{array}{c}39.4^{\mathrm{a}} \\
\pm 0.4 \\
1\end{array}$ & $\begin{array}{r}39.2^{\mathrm{a}} \\
\pm 0.63\end{array}$ & $\begin{array}{l}716.7^{\mathrm{a}} \\
\pm 14.4\end{array}$ & $\begin{array}{l}716.7^{\mathrm{a}} \\
\pm 14.4\end{array}$ & $\begin{array}{l}761.4^{\mathrm{a}} \\
\pm 63.7\end{array}$ \\
\hline Radiation & $\begin{array}{c}4.6^{\mathrm{e}} \\
\pm 0.38\end{array}$ & $\begin{array}{l}3.92^{c} \\
\pm 0.39\end{array}$ & $\begin{array}{c}3.92^{\mathrm{c}} \\
\pm 0.3 \\
9\end{array}$ & $\begin{array}{l}12.36^{\mathrm{c}} \\
\pm 0.44\end{array}$ & $\begin{array}{l}5.98^{\mathrm{e}} \\
\pm 0.10\end{array}$ & $\begin{array}{l}6.68^{d} \\
\pm 0.47\end{array}$ & $\begin{array}{c}36.9^{\mathrm{a}} \\
\pm 0.4 \\
8\end{array}$ & $\begin{array}{c}18.06 \\
c \\
\pm 0.8 \\
2\end{array}$ & $\begin{array}{r}28.5^{b} \\
\pm 3.72\end{array}$ & $\begin{array}{r}46.8^{b} \\
\pm 20.1\end{array}$ & $\begin{array}{l}139.2^{\mathrm{e}} \\
\pm 18.2\end{array}$ & $\begin{array}{c}385.6^{b} \\
\pm 33.4\end{array}$ \\
\hline R+MNPs & $\begin{array}{c}4.9^{\mathrm{e}} \\
\pm 0.28\end{array}$ & $\begin{array}{l}6.93^{\text {ab }} \\
\pm 0.06\end{array}$ & $\begin{array}{c}6.98^{a} \\
b \\
\pm 0.0 \\
6 \\
\end{array}$ & $\begin{array}{c}12.83^{b} \\
c \\
\pm 0.08\end{array}$ & $\begin{array}{l}10.94^{b} \\
\pm 0.88\end{array}$ & $\begin{array}{l}11.15^{c} \\
\pm 0.76\end{array}$ & $\begin{array}{c}37.9^{\mathrm{a}} \\
\pm 0.4 \\
8\end{array}$ & $\begin{array}{c}25.6^{6} \\
\pm 0.8 \\
2\end{array}$ & $\begin{array}{c}33.5^{\mathrm{ab}} \\
\pm 1\end{array}$ & $\begin{array}{c}69.4^{b} \\
\pm 13.7 \\
2\end{array}$ & $\begin{array}{l}501.4^{\mathrm{d}} \\
\pm 24.0\end{array}$ & $\begin{array}{c}642.8^{\mathrm{a}} \\
\pm 162 . \\
0\end{array}$ \\
\hline $\begin{array}{c}\text { R+ } \\
\text { CURMNP } \\
\text { s } \\
\end{array}$ & $\begin{array}{l}5.26^{\mathrm{de}} \\
\pm 0.33\end{array}$ & $\begin{array}{l}7.06^{a} \\
\pm 0.31\end{array}$ & $\begin{array}{c}7.06^{\mathrm{a}} \\
\pm 0.3 \\
1\end{array}$ & $\begin{array}{l}13.3^{\mathrm{ab}} \\
\pm 0.11\end{array}$ & $\begin{array}{l}12.5^{\mathrm{ab}} \\
\pm 0.31\end{array}$ & $\begin{array}{c}13.22^{\mathrm{a}} \\
\mathrm{b} \\
\pm 0.13\end{array}$ & $\begin{array}{c}39.1^{\mathrm{a}} \\
\pm 0.2 \\
5\end{array}$ & $\begin{array}{c}27.6^{b} \\
\pm 2.5 \\
2\end{array}$ & $\begin{array}{r}37.6^{\mathrm{a}} \\
\pm 1.55\end{array}$ & $\begin{array}{l}92.6^{b} \\
\pm 20.8\end{array}$ & $\begin{array}{c}606.8^{\mathrm{c}} \\
\pm 4.4\end{array}$ & $\begin{array}{c}668.8^{a} \\
\pm 81.4\end{array}$ \\
\hline
\end{tabular}

Data are presented as means \pm S.E

S.E.: Standard Error

Means values within the same column carrying different superscript letters are significantly different $(\mathbf{P}<0.05)$ 

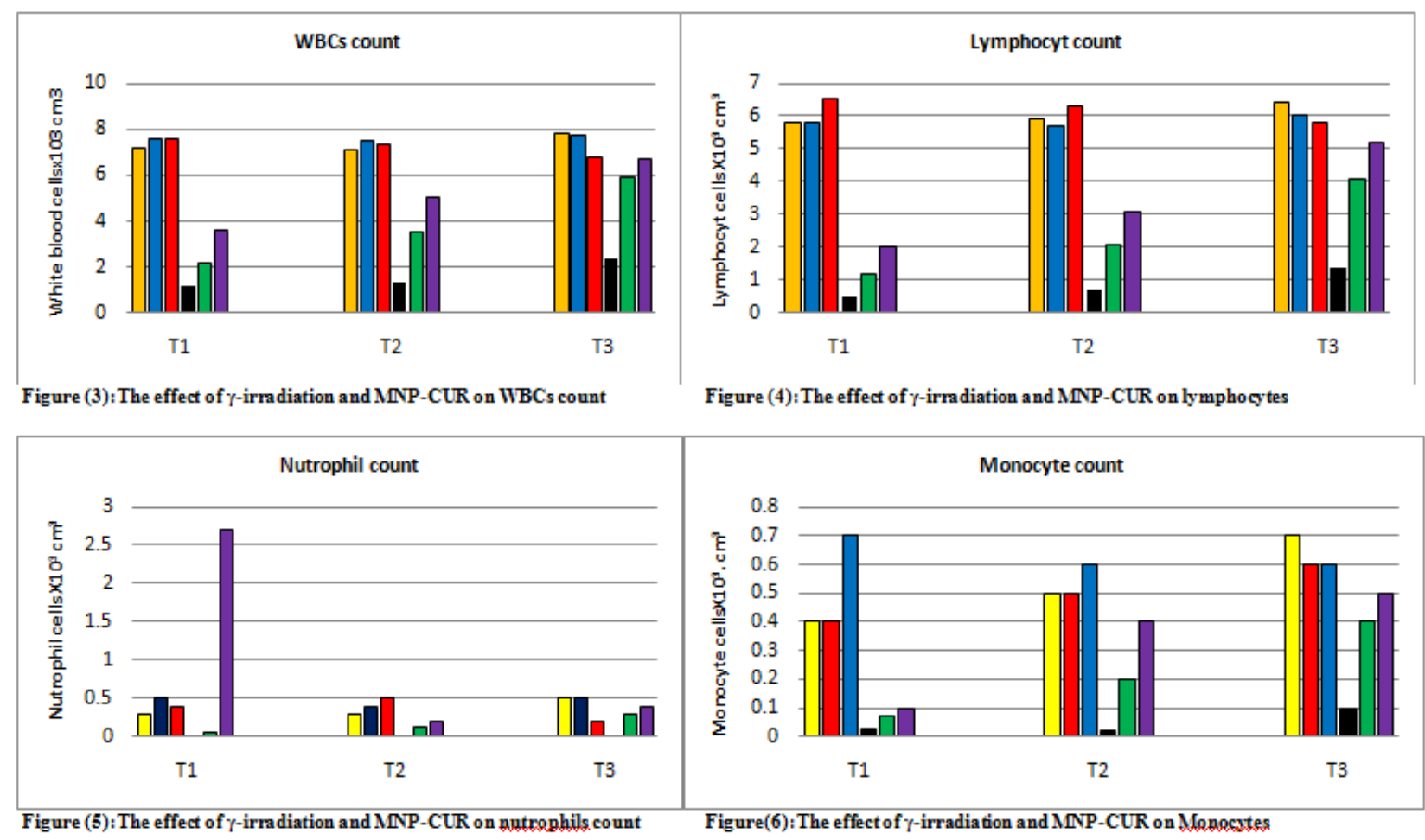

Figure (5): The effect of $\gamma$-irradiation and MNP-CUR on putrophils count

Figure(6): The effect of $\gamma$-irradiation and MNP-CUR on Mopecstes

\begin{tabular}{|llllll}
\hline$\square$ control & $\square$ MNPs & $\square$ NPs-CUR & $\mathbf{a}$ irradiation & $\square \mathrm{R}+\mathrm{MNPs}$ & $\square \mathrm{R}+\mathrm{MNPs}$-CUR \\
\hline
\end{tabular}
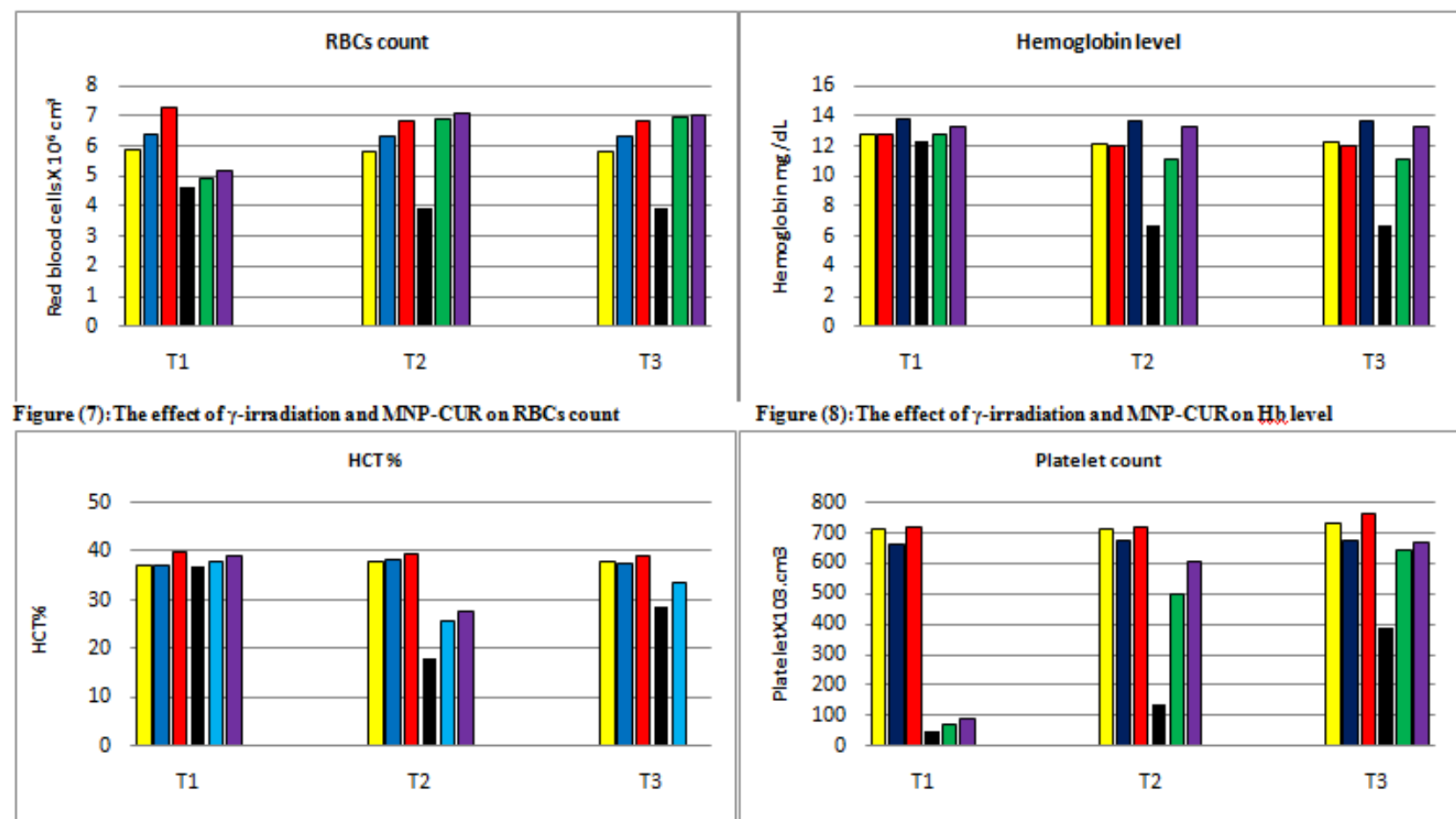

Figure (8): The effect of $\gamma$-irradiation and MNP-CUR on Hb level

Figure (9): The effect of $\gamma$-irra diation and MNP-CUR on HCT $\%$

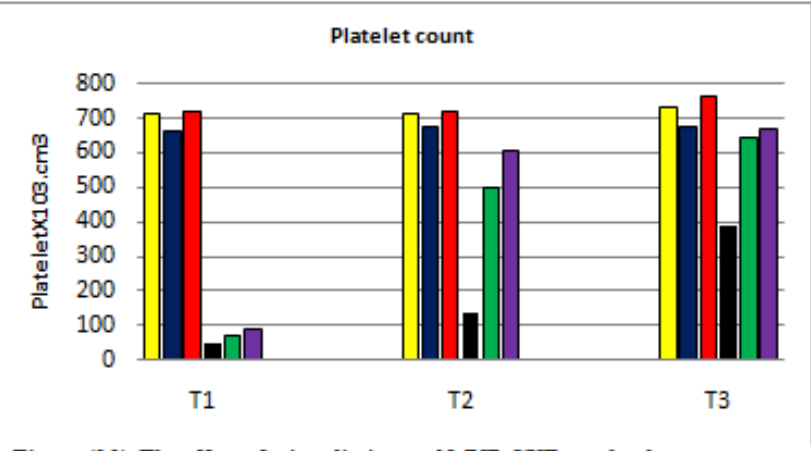

Figure (10): The effect of $\gamma$-irradiation and MNP-CUR on platelet count

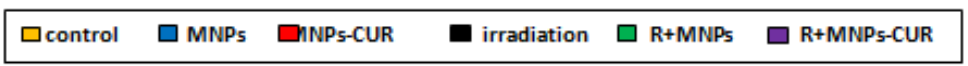

Arab J. Nucl. Sci. \& Applic. Vol. 52, No.3 (2019) 
Table (3):the effect of $\gamma$-irradiation and MNPs-CUR on L-Malnodialdehyde, Glutathione and catalase

\begin{tabular}{|c|c|c|c|c|c|c|c|c|c|}
\hline \multirow{3}{*}{$\begin{array}{c}\text { Parameter } \\
\text { Group }\end{array}$} & \multicolumn{9}{|c|}{ Post irradiation } \\
\hline & \multicolumn{3}{|c|}{ MAD } & \multicolumn{3}{|c|}{ GSH } & \multicolumn{3}{|c|}{ CAT } \\
\hline & $\mathbf{T}_{1}$ & $\mathbf{T}_{2}$ & $\mathbf{T}_{\mathbf{3}}$ & $\mathbf{T}_{1}$ & $\mathbf{T}_{2}$ & $\mathbf{T}_{3}$ & $\mathbf{T}_{1}$ & $\mathbf{T}_{2}$ & $\mathbf{T}_{3}$ \\
\hline Control & $\begin{array}{r}33.3^{\mathrm{e}} \\
\pm 0.73\end{array}$ & $\begin{array}{c}33.8^{\mathrm{e}} \\
\pm 0.55\end{array}$ & $\begin{array}{l}\mathbf{3 3 . 7 3}^{\mathrm{e}} \\
\pm \mathbf{0 . 5 3}\end{array}$ & $\begin{array}{c}33.73^{\mathrm{a}} \\
\pm 0.91\end{array}$ & $\begin{array}{c}\mathbf{3 3 . 0 3}^{\text {ab }} \\
\pm \mathbf{0 . 3 7}\end{array}$ & $\begin{array}{c}3^{3.23}{ }^{\text {ab }} \\
\pm 0.41\end{array}$ & $\begin{array}{l}4.75^{b} \\
\pm 0.15\end{array}$ & $\begin{array}{l}4.75^{b} \\
\pm 0.38\end{array}$ & $\begin{array}{l}4.74^{b} \\
\pm 0.31 \\
\end{array}$ \\
\hline MNPs & $\begin{array}{l}32.0^{\text {ef }} \\
\pm 0.58\end{array}$ & $\begin{array}{c}\text { 31.1 } \\
\pm 0.38\end{array}$ & $\begin{array}{r}\mathbf{3 1 . 2} \mathbf{f}^{\mathrm{f}} \\
\pm \mathbf{0 . 3 7}\end{array}$ & $\begin{array}{c}29.07^{b} \\
\pm 0.52\end{array}$ & $\begin{array}{c}29.56^{c} \\
\pm 0.54\end{array}$ & $\begin{array}{c}29.58^{c} \\
\pm 0.53\end{array}$ & $\begin{array}{l}4.98^{\mathrm{ab}} \\
\pm 0.08\end{array}$ & $\begin{array}{l}5.12^{b} \\
\pm 0.18\end{array}$ & $\begin{array}{r}5.14^{b} \\
\pm 0.17\end{array}$ \\
\hline CURMNPs & $\begin{array}{c}30.8^{f} \\
\pm 0.73\end{array}$ & $\begin{array}{l}3^{30.13}{ }^{\mathrm{f}} \\
\pm 0.44\end{array}$ & $\begin{array}{l}30.15^{\mathrm{f}} \\
\pm 0.45\end{array}$ & $\begin{array}{c}33.67^{\mathrm{a}} \\
\pm 0.88\end{array}$ & $\begin{array}{l}34.24^{\mathrm{a}} \\
\pm 0.33\end{array}$ & $\begin{array}{c}34.28^{\mathrm{a}} \\
\pm 0.38\end{array}$ & $\begin{array}{l}5.33^{\mathrm{a}} \\
\pm 0.37\end{array}$ & $\begin{array}{r}6.15^{\mathrm{a}} \\
\pm 0.08\end{array}$ & $\begin{array}{r}6.16^{\mathrm{a}} \\
\pm 0.08 \\
\end{array}$ \\
\hline Radiation & $\begin{array}{r}68.4^{\mathrm{a}} \\
\pm 0.72\end{array}$ & $\begin{array}{l}65.47^{\mathrm{a}} \\
\pm 0.88\end{array}$ & $\begin{array}{l}65.46^{\mathrm{a}} \\
\pm 0.86\end{array}$ & $\begin{array}{l}10.41^{\mathrm{e}} \\
\pm 0.30\end{array}$ & $\begin{array}{l}10.41^{\mathrm{e}} \\
\pm 0.30\end{array}$ & $\begin{array}{l}10.33^{\mathrm{e}} \\
\pm 0.32\end{array}$ & $\begin{array}{r}1.07^{\mathrm{e}} \\
\pm 0.06\end{array}$ & $\begin{array}{c}0.98^{\mathrm{e}} \\
\pm 0.02\end{array}$ & $\begin{array}{c}0.97^{\mathrm{e}} \\
\pm 0.03\end{array}$ \\
\hline R+MNPs & $\begin{array}{r}68.4^{\mathrm{a}} \\
\pm 0.72\end{array}$ & $\begin{array}{l}49.30^{b} \\
\pm 0.35\end{array}$ & $\begin{array}{c}49.28^{b} \\
\pm 0.34\end{array}$ & $\begin{array}{l}24.10^{d} \\
\pm 0.55\end{array}$ & $\begin{array}{l}24.10^{d} \\
\pm 0.55\end{array}$ & $\begin{array}{l}24.28^{d} \\
\pm 0.54\end{array}$ & $\begin{array}{r}2.23^{d} \\
\pm 0.12\end{array}$ & $\begin{array}{l}2.83^{d} \\
\pm 0.12\end{array}$ & $\begin{array}{l}2.84^{d} \\
\pm 0.12\end{array}$ \\
\hline R+ CURMNPs & $\begin{array}{l}41.6^{\mathrm{d}} \\
\pm 0.68\end{array}$ & $\begin{array}{l}41.6^{\mathrm{d}} \\
\pm 0.68\end{array}$ & $\begin{array}{l}41.55^{\mathrm{d}} \\
\pm 0.68\end{array}$ & $\begin{array}{l}26.40^{c} \\
\pm 0.83\end{array}$ & $\begin{array}{l}27.30^{\mathrm{d}} \\
\pm \mathbf{0 . 5 1}\end{array}$ & $\begin{array}{c}27.32^{\mathrm{d}} \\
\pm 0.5\end{array}$ & $\begin{array}{r}2.97^{\mathrm{c}} \\
\pm 0.15\end{array}$ & $\begin{array}{c}4.06^{c} \\
\pm 0.10\end{array}$ & $\begin{array}{l}4.06^{c} \\
\pm 0.09\end{array}$ \\
\hline
\end{tabular}

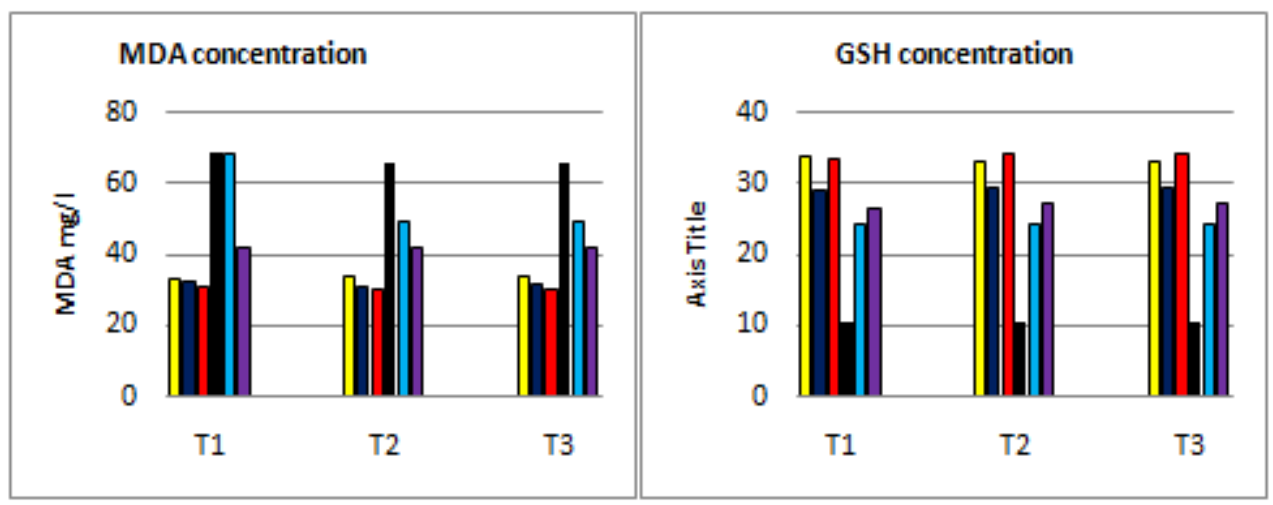

Figure (11): The effect of $\gamma$-irradiation and MNPs- Figure (12): The effect of $\gamma$-irradiation and MNPsCUR on MDA CUR on GSH

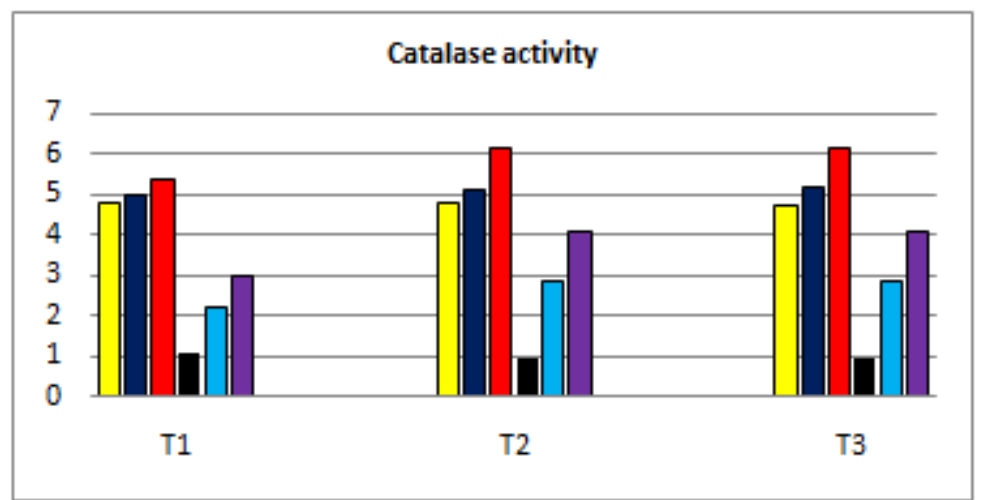

Figure (13): The effect of $\gamma$-irradiation and MNPs-CUR on CAT

$\square$ control $\square$ MNPs $\square$ MNPs-CUR $\square$ irradiation $\square$ R+MNPs $\square$ R+MNPs-CUR




\section{Discussion}

Bioactive substances are found to improve in efficacy when loaded in nanoparticles such as MNPs. This improvement is directly influenced by the efficiency of loading of the bioactive substance in nanoparticles [27]. Although CUR is known to have antioxidant and anti-inflammatory activity, its use is limited because of its low bioavailability and poor pharmacokinetics [28]. Therefore, a CURMNPsnanosystem was developed to increase the bioavailability, pharmacokinetics and subsequently improve the therapeutic efficacy of CUR. Total body exposure to $I R$, as a cytotoxic agent, is generally assumed to cause damage to the hematopoietic cell system resulting in effects that contribute to the hematopoietic syndrome [29]. The results in the present study showed that WBCs were found to be most sensitive to $\gamma$ - ray irradiation which is in agreement with the results of Sanzari, et al. (2013) [2]. The present results are also comparable with those of Thrall et al., (2013)[30], who detected a statistically significant reduction in WBC counts at $24 \mathrm{~h}$ (one day) postirradiation for animals at 6Gy gamma irradiation, and Seed et al.,(2002), who reported that the lymphocytes, Neutrophils, Monocytes showed decrease within the first days after irradiation while platelet count declined 5-10 days post irradiation[31]. The continuous radiation induced excess cell losses in the stem and precursor cell pools, proposed by Graessle (2002), may explain the slow recovery in WBCs counts throughout time of the study [32].

A dramatic elevation of the ROS was proposed by Davis, et al., (2001) to induce apoptotic pathways in the cells as a result of the sever imbalance in the redox potential between ROS and endogenous thiol buffers[33].

Glutathione acts synergistically with other endogenous antioxidants and also acts as a cofactor with the enzyme glutathione peroxidase to scavenge free radicals, and detoxify xenobiotics [34]. Thus, the depletion of GSH contents after exposure to $\gamma$-radiation may be due to reaction of reduced form of Glutathione GSH with free radicals resulting in the formation of thiol radicals that react to produce oxidized form of Glutathione GSSG as reported earlier by Navarro, et al. in 1997 [35].

The intraperitoneal administration of MNP-CUR significantly enhanced the endogenous GSH and reduced the MDA levels in the liver of the irradiated rats which may be attributed to increased bioavailability of CUR.This may be due to administration as CUR-MNP resulting in more efficient anti antioxidant action of CUR that subsequently reduced the overwhelming load of ROS, caused by $\gamma$-irradiation, on the endogenous antioxidant systems including the glutathione peroxidase and CAT systems.

\section{Conclusions}

The efficacy of CUR-MNP to reduce the cellular damage induced by ionizing radiation was studied. MNP-CUR ameliorates the oxidative damage of $\gamma$ radiation. CUR-MNP provides a radio-resistance effect to bone marrow cells in animals that received $\gamma$-radiation. The use of commercial CUR may be limited due its poor oral bioavailability and solubility in plasma. The present study, with these naturally antioxidant loaded nanoparticles, provides an extended window for protection against sublethal-dose irradiation. It could be concluded that CUR-MNPs can be used as a radio protective agent to the professional radiation and remediation workers who may be exposed to low levels of ionizing radiation, and especially if it is administered after irradiation for therapeutic purposes.

\section{References}

1-El-shanshoury H. , El-shanshoury G., and Abaza A., (2016) Evaluation of low dose ionizing radiation effect onsome blood components in animal model. J. of radiation research and applied Sciences. 282-293.

2- Sanzari J. K., Wan X. S., Krigsfeld G. S., Wroe A. J., Gridley D. S., and Kennedy A. R. (2013). The effects of $\mathrm{g}$ amma and proton radiation exposure on hematopoietic cell counts in the ferret model. Gravitational and space Research, 1(1),79-94.

3- Maks C. J., Wan X. S., Ware J. H., Romero-Weaver A. L., Sanzri J. K., and Wilson J. M., (2011). Anlysis of white blood cell counts in mice after gamma- or proton- radiation exposure. Radiation Research, 176(2), 170-176.

4-Kennedy A. R., Romero-Weaver A.L., Wan X.S., Diffenderfer E.S., and Lin L., (2013). Kinetic of neutrophils in mice exposed to radiation and / granulocyte colony stimulating factor treatment . Radiation Research, 180(2), 177-188.

5-Pratheeshkumar P., and Girija K (2010). Protective role of VernoniaCinerea L. against gamma radiationinduced immunosuppression and oxidative stress in 
mice. J. Human and experimental toxicology 30(8) 1022-1038.

6- Adour M. I., and El-Syyad H., (2012). The protective effect of oyster Mushroom (Pleurtuspulmonarus) aginstsublethal gamma radiation dose in rats. Arab j. of nuclear sci. and Applic, (45): 234-255.

7-Gupta A. K., and Gupta M., (2005). Synthesis and surface engineering of iron oxide nanoparticles for biomedical applications. Biomaterials J., (18): 3995 4021.

8- Thorek D. L., Chen A. K., Czypryna J., and Tsourkas A., (2006). Superparamagnetic iron oxide nanoparticles probes for molecular imaging Ann Biomed Eng., (34): 23-38.

9-Sun C., Lee J., and Zhang M., (2008). Magentic nanoparticles in NMR imaging and drug delivery. Adv Drug Deliv Rev60, (11): 1252-1265.

10-Shubayev V. I., Pisanic T. R., and Jin S., (2009). Magnetic nanoparticles for theragnostic. Adv. Drug Deliv. Rev., (61): 467-477.

11-Bhasskar S., Tian F., Stoeger T., Kreyling W., Fuenteet J. M. de la, Grazu V., Brom P., Estrada G., NtziachritosV.,andRazansky D., (2010). Multifunctional nanocarriers for diagnostics, durg delivery and targeted treatment across blood-brain barrier: Perspectives on tracking and neuroimaging. Part fibreToxicol, (7): 1-3.

12-Brigitta S., PhD Thesis, (2012). Iron oxide nanoparticles and their toxicological effects; in vivo and in vitro studies. Department of Public Health Faculty of Medicine University of Szeged .

13-Joe B., Vijaykumar M., and Lokesh B.R.,(2004). Biological properties of curcumin-cellular and molecular mechanisms of action, Critical reviews in food Science and Nutrition, (44),97-111.

14-Bayomi S. M., El-kashef H. A., El-Ashmawy M. B., Nasr M. N. A., El-Sherbeny M. A., Badria F. A., Abou-Zeid L. A., Ghaly M. A., and Abdel- Aziz N. I., (2013). Synthesis and biological evaluation of new curcumin derivatives as antioxidant as antioxidant and antitumor agents, Medicinal Chemistry Resesrch, (22).1147-1162.

15-Shen L.,Ji H-F, (2009). Insights into the inhibition of xanthine oxidase by curcumin, Bioorganic and Medicinal Chemistry Letters,(19). 5990-5993.

16-Goel A., and Aggarwal B.B., (2010), Curcumin, the golden spices from Indian saffron, is a chemosensitizer and radiosensitizer for tumors and chemoprotector and radioprotector for normal organs, Nutrition and cancer, 62, 919-930.

17- Ozgen S.C., Dokmeci D., Akpolat M., Karadag C. H., Karadag O., Gunduz H., Erbas H., Benian O., Uzal C., andTuran F.N.,(2012), The protective Effect of curcumin on ionizing Radiation- induced cataractogenesis in rats, Ballkan Medical Journal, 29, 358-363.

18-Jha N.S., Mishra S., Jha S.K., and Surolia A., (2015). Antioxidant activity and electrochemical elucidation of the enigmatic redox behavior of curcumin and its structurally modified analogues, ElectrochimicaActa,(151). 574-583.

19-Gupta S. C., Patchva S., and Aggarwal B.B., (2013). Therapeutic roles of curcumin: Lessons learned from clinical trials, The AAPS journal, (15).195-218.

20-Anand P., Kunnumakkara A.B., Aggarwal B.B., and Newman R.A.,(2007). Bioavailability of curcumin: Problems and promises, Molecular Pharmaceutics. (4). 807-818.

21-Yallapu M M, Othman SF, Curtis ET, Gupta BK,Jaggi M, and Chauhan SC, (2011), Multifunctional magnetic nanoparticles for magnetic resonance imaging and cancer therapy. Biomaterials;32(7):1890-1905.

22- Bhattarai S.R., Kc R.B., and Kim S.Y., (2008) Nhexanoyl chitosan stabilized magneticnanoparticles:implication for cellular labeling and magnetic resonance imaging. $\mathbf{J}$ Nanobiotechnology.6:1.

23-Trivelli, L. A., Ronney, H. M., \& Lai, H. T. (1971). Hemoglobin components in patients with diabetus mellitus. National England Journal of Medicine, 284, 353.

24-Pagila D. E., and Valentine W. N., (1967). Studies on the quantitative and qualitative characterization of erythrocyte glutathione peroxidase. J. Lab. Clin. Med. (1): 158-69.

25-Aebi H., (1984). Catalase in vitro. J. Methods in Enzymology. (105), 121-126.

26- Oh Kawa, H.; Ohishi, N., and Yagi , K.; (1979): Assay for lipid peroxides in animal tissues by thiobarbituric acid reaction. Anal biochem.; (2): 351-8.

27- Konwarh R., Karak N., Rai S.K., and Mukherjee A. K., (2009). Nanotechnology 20. 3621-3623.

28- Anand P., Sundaram C., Jhurani S., Kunnumakkara A.B., and Aggarwal B.B., (2008), Curcumin and cancer: an "old-age" didease with an " age- old" solution. Cancer Lett. 267(1):133-164.

29-Billings P. C., Romero-Weaver A. L., and Kennedy A. R.,(2014). Effect of gender on the radiation sensitivity of murine blood cells. Gravitational and Space Research, 2(1), 25-31.

30-Thrall K. D., Lovaglio J., Murphy M. K., Cataneo R. N., Chaturvedi A., and Mundada M., (2013). A dosedependent hematological evaluation of whole-body gamma-irradiation in the Gottingen minipig. Health Physics, 105(3), 245-252.S

31-eed T. M., Fritz T. E., Tolle D. V., and Jackson W. E., (2002). Hematopoieteic responses under protracted exposures to low daily dose gamma irradiation. Advances in Space Research, 30 (4), 945955.

32-Graessle D. H., (2002). Mathematical modeling of the blood platelet renewal system as an approach to analyzing the effects of chronic irradiation on 
haematopoiesis. The British Journal of Radiology Supplement, 26, 202-207.

33-Davis W. Jr., Ronai Z., and Tew K., D.,(2001) cellular thiols and reactive oxygen species in druginduced apoptosis.j. pharmacol. Exp.Therapeut.296,1-6.

34-Aquilano, K., Baldelli, S.,andCiriolo, M.R.(2014) Glutathione: New roles in redox signaling for an old antioxidant. Front. Pharmacol. 5,196.
35-Navarro, J., Obrador, E., Pellicer,J.A.,Aseni, M., Vina,J., and Estrela,M. (1997). Blood glutathione as an index of radiation -induce oxidative stress in mice and humans. Free Radic.Biol.Med. 22, 1203-1207. 